\title{
Comparação de Desempenho e Consumo de Energia de Aplicações Paralelizadas com OpenMP taskloop e parallel for
}

\author{
Luan Pereira ${ }^{1}$, Sandro Matheus V. N. Marques ${ }^{1}$, Fábio D. Rossi ${ }^{2}$, \\ Marcelo C. Luizelli ${ }^{1}$ e Arthur F. Lorenzon ${ }^{1}$ \\ ${ }^{1}$ Laboratório de Otimização de Sistemas - Universidade Federal do Pampa \\ Campus Alegrete (UNIPAMPA) - Alegrete - RS - Brasil \\ ${ }^{2}$ Instituto Federal Farroupilha - Campus Alegrete - Alegrete - RS - Brasil \\ luanvargas.aluno@unipampa.edu.br
}

\begin{abstract}
Resumo. A biblioteca OpenMP detém diretivas que possuem o mesmo propósito, porém com funcionalidade diferente. Um exemplo disso é a diretiva \#pragma omp for e a \#pragma omp taskloop. Neste trabalho apresentamos uma análise de tempo de execução e consumo energético de ambas diretivas. Nós mostramos que a utilização da diretiva parallel for é até $42 \%$ mais rápida e consome 27,21\% menos energia que a implementação com taskloop. ${ }^{1}$
\end{abstract}

\section{Introdução}

O uso de diferentes técnicas de programação paralela tem sido cada vez mais recorrente pela comunidade de alto desempenho [Lorenzon and Beck Filho 2019]. Estas técnicas são normalmente aferidas à bibliotecas de programação paralela, como por exemplo, o Open MultiProcessing (OpenMP). A biblioteca OpenMP possui conjuntos de diretivas que facilitam a exploração do paralelismo. Com isso é possível encontrar diretivas que possuem objetivos semelhantes com funcionalidades diferentes, como por exemplo, \#pragma omp taskloop e \#pragma omp for, utilizadas para a exploração de paralelismo em laços.

Na exploração de paralelismo com o uso de taskloop, tasks são criadas para um intervalo (chamado de chunk) de iterações do laço e são armazenadas em uma estrutura (i.e., bag of tasks) [Bronis R. de Supinski 2019]. Por outro lado, quando se usa o parallel for, as iterações do laço são divididas em chunks através de um scheduler definido a priori, que serão atribuídos para cada uma das threads. Neste sentido, muito embora ambas as estratégias têm o mesmo objetivo, é importante avaliar o comportamento dessas diretivas em relação ao desempenho e consumo de energia.

Diferentes trabalhos têm avaliado o uso das diretivas para exploração de paralelismo em laços do OpenMP. X. Teruel et al. [Teruel et al. 2013] propõe a diretiva taskloop e comparam o seu desempenho com outras implementações de laços paralelos. A. Podobas et al. [Podobas and Karlsson 2016] avaliam a escalabilidade e desempenho de ambas as diretivas, mostrando que o taskloop pode ser até $3.2 \%$ mais rápido que o parallel for. A. Rico et al. [Rico et al. 2019] analisam o desempenho e escalonamento de threads ao reimplementar um benchmark utilizando a diretiva taskloop. $\mathrm{O}$ autor conclui que a execução é até 30\% mais rápida com a diretiva taskloop quando comparada com o parallel-for.

\footnotetext{
${ }^{1}$ Este trabalho foi parcialmente financiado pela FAPERGS nos projetos 19/2551-0001224-1 e 19/25510001689-1, CNPq PIBIC e FAPERGS PROBIC.
} 
Diferentemente dos trabalhos já realizados, apresentamos uma avaliação das diretivas taskloop e parallel for em aplicações paralelas considerando o desempenho e o consumo de energia. Através da execução de quatro aplicações, mostramos que a implementação com parallel for é até $42 \%$ mais rápida e consome $27,21 \%$ menos energia que a implementação com taskloop.

\section{Programação Paralela com OpenMP}

O OpenMP é uma biblioteca para programação em memória compartilhada em $\mathrm{C} / \mathrm{C}++$ e FORTRAN, que consiste de um conjunto de diretivas de compiladores, bibliotecas de funções e variáveis de ambientes [Bronis R. de Supinski 2019]. O paralelismo é explorado através da inserção de diretivas no código sequencial que informam ao compilador como e quais partes do código devem ser executadas em paralelo. Duas destas diretivas compreendem a \#pragma omp taskloop e \#pragma omp for, que realizam a distribuição da carga de trabalho em uma região paralela. As principais características de cada uma delas são descritas abaixo.

Taskloop: É um construtor gerador de tasks. Quando é invocado, as iterações de um laço são particionadas em tasks para a execução paralela. A divisão dos dados de cada task é criada de acordo com as cláusulas de compartilhamento de dados (i.e., private e shared). As tasks são criadas e armazenadas em uma estrutura interna do OpenMP chamada bag of tasks [Bronis R. de Supinski 2019] até sua execução. Por padrão, a execução das tasks é feita de acordo com a disponibilidade das threads no Sistema Operacional.

Laços paralelos: Quando o parallel for é invocado, as iterações são dividas em uma série de chunks. Para cada chunk, é determinada qual thread será atribuída a sua execução. Essa atribuição ocorre de acordo com o tipo de scheduler definido na variável de ambiente \$OMP_SCHEDULE. Contudo, o tipo scheduler pode ser alterado em código por meio do uso da cláusula (schedule) em conjunto com o parallel for. Os schedulers são divididos em quatro tipos: dynamic; guided; auto; e runtime. Por padrão, o compilador GCC 9.2 define o scheduler em dynamic. Neste caso, cada thread executa um chunk de iterações, e então, demanda um novo chunk, até que todas as iterações estejam finalizadas [Bronis R. de Supinski 2019].

\section{Metodologia}

Os experimentos foram realizados no processador AMD Ryzen 9 3900X de 12 núcleos com o Sistema Operacional Ubuntu, kernel v.4.15.0. Este processador possui tecnologia Simultaneous Multi-Threading (SMT), podendo executar até 24 threads. Cada núcleo físico possuí uma memória cache L1 de dados e instruções de $32 \mathrm{~KB}$ cada e uma cache L2 de 512KB. Cada grupo de três núcleos compartilham uma memória cache L3 de 16MB. O sistema como um todo possui 32 GB de memória principal.

Foram escolhidas quatro aplicações escritas em $\mathrm{C}$ e paralelizadas com OpenMP, com diferentes domínios: fast fourier transform (FFT), Jacobi (JA), Poisson (PO) e STREAM (ST). As aplicações foram executadas no compilador GCC 9.2.0 com a flag de otimização -O3 e com as entradas padrões dos benchmark escolhidos. Consideramos a versão do OpenMP 5.0. Para cada uma das aplicações foram feitas execuções com diferentes números de threads: 1, 2, 4, 8, 12 e 24. O DVFS governor estava configurado em ondemand. Este governor, ajusta a frequência de cada núcleo de acordo com a carga 
de trabalho da aplicação. Foram feitas cinco execuções para cada aplicação e número de threads. O tempo de execução de cada aplicação foi obtido através da função do OpenMP omp_get_wtime enquanto que o consumo de energia do processador e sistema de memória diretamente do hardware através da biblioteca RAPL (running average power limit).

\section{Resultados}

A Figura 1 apresenta o tempo de execução das aplicações executadas em função do número de threads, onde considerando a média de todas as aplicações utilizando parallel for, foi $42 \%$ menor em relação as aplicações utilizando a diretiva taskloop. No entanto, há casos em que essa diferença é menor, como por exemplo, nas aplicações FFT (Figura 1.a) e STREAM (Figura 1.D), onde a diferença é de apenas $2 \%$ e $0.1 \%$, respectivamente. Este comportamento acontece pois ambas as aplicações fazem muitos acessos à memória durante a execução, o que gera um sobrecusto maior para a aplicação do que a diferença entre threads e tasks [Lorenzon et al. 2018]. Por fim, nas aplicações JA (Figura 1.b) e PO (Figura 1.c) a diferença no tempo de execução foi de $55.8 \%$ e 52\%, respectivamente, assim apresentando um impacto maior na média geral de $42 \%$.

Na Figura 2 pode-se observar o consumo de energia das aplicações em função do número de threads que foram executadas. Considerando a média de todas as aplicações, o uso da diretiva task loop gerou um aumento de $27 \%$ no consumo de energia. Por outro lado, em algumas aplicações o consumo de energia foi muito semelhante, como por exemplo, na aplicação FFT (Figura 2.a) e STREAM (Figura 2.d). Já a aplicação JA (Figura 2.b) implementada com for obteve uma média de consumo de energia $46 \%$ menor em comparação com a versão implementada com taskloop. Por fim, a PO (Figura 2.c) implementada com for mediu-se um consumo médio $42 \%$ menor do que na com taskloop.

Considerando os resultados obtidos nas execuções, a diretiva parallel for mostrou um melhor desempenho e eficiência energética em relação ao taskloop. Contudo, os benefícios do uso do parallel for se mostram mais presentes em aplicações cuja a dependência dados é estática. Sendo assim, em aplicações que envolvem algoritmos com dados dinâmicamente dependentes o taskloop se mostra mais adequado. Isto ocorre, por

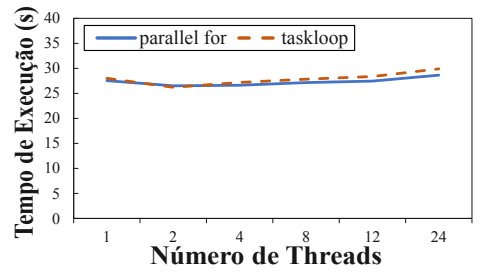

(a) FFT

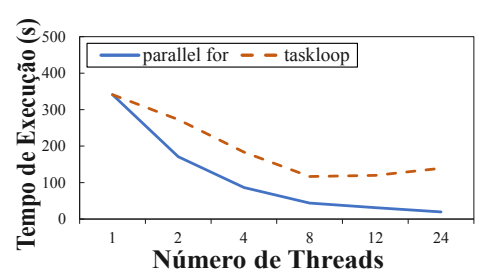

(c) $\mathrm{PO}$

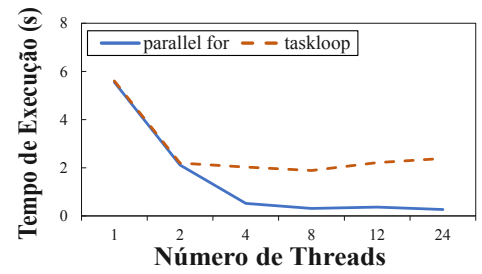

(b) JA

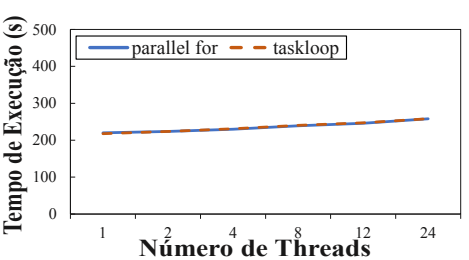

(d) $\mathrm{ST}$

Figura 1. Tempo de execução de cada aplicação para cada configuração 


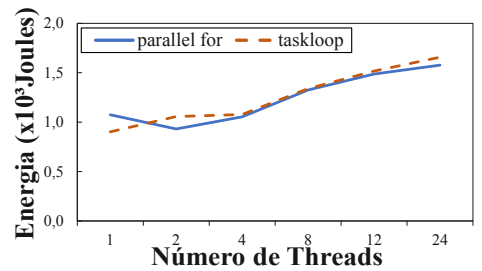

(a) FFT

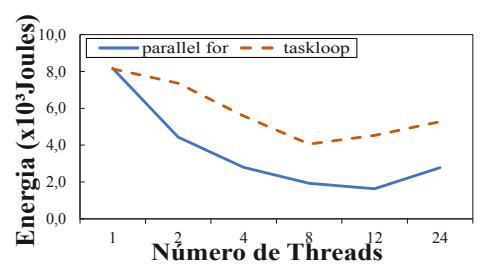

(c) $\mathrm{PO}$

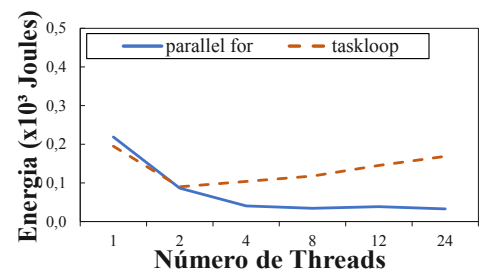

(b) JA

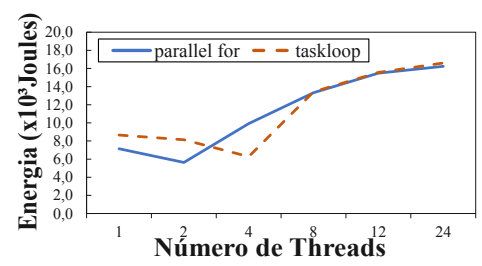

(d) ST

Figura 2. Consumo de energia de cada aplicação para cada configuração

que o uso do taskloop permite melhor balanceamento de carga entre as threads [Teruel et al. 2013] [Podobas and Karlsson 2016] [Rico et al. 2019].

\section{Conclusão}

Este trabalho realizou uma comparação do uso de duas diferentes diretivas de laços paralelos do OpenMP, o taskloop e parallel for. Mostrou-se que o uso da diretiva parallel for apresenta melhor desempenho, consumo de energia e escalabilidade em comparação ao taskloop. Como trabalhos futuros, pretende-se expandir o ambiente de execução para compreender outras aplicações e métricas.

\section{Referências}

Bronis R. de Supinski, M. K. (2019). Openmp technical report 8: Version 5.1 preview. Technical report, OpenMP Architecture Review Board.

Lorenzon, A. F. and Beck Filho, A. C. S. (2019). Parallel Computing Hits the Power Wall Principles, Challenges, and a Survey of Solutions. Springer.

Lorenzon, A. F., De Oliveira, C. C., Souza, J. D., and Beck, A. C. S. (2018). Aurora: Seamless optimization of openmp applications. IEEE Transactions on Parallel and Distributed Systems, 30(5):1007-1021.

Podobas, A. and Karlsson, S. (2016). Towards unifying openmp under the task-parallel paradigm. In International Workshop on OpenMP, pages 116-129. Springer.

Rico, A., Barrera, I. S., Joao, J. A., Randall, J., Casas, M., and Moretó, M. (2019). On the benefits of tasking with openmp. In International Workshop on OpenMP, pages 217-230. Springer.

Teruel, X., Klemm, M., Li, K., Martorell, X., Olivier, S. L., and Terboven, C. (2013). A proposal for task-generating loops in openmp. In International Workshop on OpenMP, pages $1-14$. Springer. 